\title{
Facteurs communautaires influençant la déperdition Penta1-Penta3 des enfants de 0-11 mois dans le district sanitaire de la Commune IV, Bamako
}

\section{Community factors influencing the Penta1-Penta3 loss of children 0-11 months in the Commune IV, Bamako}

\author{
A. Témé ${ }^{1,2}$, K. Keita ${ }^{2}$, M.S. Konaké ${ }^{3}$, S. Tounkara ${ }^{1}$, D. Simpara ${ }^{1}$, O. Sangho ${ }^{1,4}$, F. Diawara ${ }^{1,4}$, F. Sangho ${ }^{1,4}$, H. Sangho ${ }^{1}$
}

1. Département d'Enseignement et de Recherche en Santé Publique (DERSP), FMOS, USTTB

2. Direction Régionale de la Santé de Kidal

3. Centre de Santé de Référence de la Commune VI, Bamako, Mali

4. Faculté de Pharmacie

\section{Résumé}

Introduction: La vaccination est une mesure efficace pour prévenir les maladies infectieuses chez les enfants. L'objectif de cette recherche était d'étudier les facteurs communautaires influençant la déperdition entre le Pentavalent1 et le Pentavalent2 chez les enfants de 0-11 mois dans le district sanitaire de la commune IV (Bamako). Matériels et Méthodes: Une étude transversale, descriptive et analytique a été menée. Selon la formule de Schwartz 360 tuteurs d'enfants de 0 à 11 mois ont été sélectionnés par sondage en grappes à deux degrés et 20 agents de santé impliqués dans les activités de vaccination sélectionnés par un choix raisonné ont été interviewé à l'aide d'un questionnaire. Une analyse de régression logistique multi-variée a été faite pour les variables ayant obtenu une valeur $p<0,05$ à l'analyse bi-variée en utilisant le logiciel SPSS 21 . Résultats: Le taux de déperdition Pentavalent1Pentavalent3 était de $24,44 \%$. La régression logistique multi-variée a montré que la méconnaissance des avantages de la vaccination $(p=0,007)$, la méconnaissance des maladies cibles du Penta $(p=0,002)$, le long temps d'attente $(p=0,023)$ et l'indisponibilité des tuteurs $(p=0,046)$ étaient significativement associés au taux de déperdition Penta1-Penta3 des enfants. Conclusion: Le taux de déperdition était en deçà des normes requises (taux < $10 \%)$. Son amélioration nécessite la mise à l'échelle de la stratégie "Atteindre Chaque District», "Atteindre Chaque Enfant », l'information et la sensibilisation de la communauté sur les bienfaits et les avantages de la vaccination

Mots Clés: déperdition, pentavalent, facteurs, communautaires, Commune IV, Bamako.

\footnotetext{
Abstract

Introduction: Vaccination is an effective measure to prevent infectious diseases in children. The objective of this research was to study the community factors influencing the loss of Pentavalent1 and Pentavalent2 in children aged 0-11 months in the health district of Commune IV (Bamako). Materials and Methods: A cross-sectional, descriptive and analytical study was conducted. According to Schwartz's formula 360 tutors of
}

children from 0 to 11 months were selected by two-stage cluster survey and 20 health workers involved in the vaccination activities selected by a reasoned choice were interviewed using a questionnaire. A multivariate logistic regression analysis was performed for the variables that obtained a p-value of 0.05 on the bi-varied analysis using the SPSS 21 software. Results: The Pentavalent1Pentavalent3 loss rate was $24.44 \%$. The multi-varied logistic regression showed that the ignorance of the benefits of the vaccination ( $p=0.007)$, the lack of knowledge of Penta target diseases $(p=0.002)$, the long waiting time $(p=0.023)$ and the unavailability guardians $(p=0.046$ ) were significantly associated with the Penta1Penta3 loss rate of children. Conclusion: the rate of wastage was below the required standards (rate $<10 \%$ ). Its improvement requires scaling the "Reach Every District", "Reach Every Child" strategy, community education and awareness about the benefits and benefits of immunization.

Key words: pentavalent, loss, community, factors, Commune IV, Bamako.

\section{Introduction}

La vaccination est l'une des mesures les plus efficaces pour prévenir la mortalité, la morbidité et les complications liées aux maladies infectieuses chez les enfants [1]. Après le succès du programme d'éradication de la variole, l'Organisation Mondiale de la Santé (OMS) lors de sa 27ème assemblée mondiale en 1974 a lancé un vaste programme d'immunisation, contre les principales maladies évitables par la vaccination des enfants [2]. En 2016 , environ $86 \%$ (116,5 millions) des nourrissons dans le monde ont eu les 3 doses du vaccin antidiphtériqueantitétanique-anticoquelucheux (DTC) et 130 pays avaient atteint une couverture DTC3 d'au moins 90\% [3]. Cette couverture mondiale était inférieure à $5 \%$ en 1974 [4]. Cependant elle était inférieure à $50 \%$ en 2016 dans certains pays africains, à savoir, la Guinée équatoriale, le Nigéria, la République centrafricaine, la Somalie, le Soudan du Sud et le Tchad [5].

Dans le but d'améliorer la qualité de vie des populations à travers la mise en œuvre de la stratégie de lutte contre la pauvreté, le gouvernement du Mali, dans sa stratégie sectorielle de santé s'est fixé l'objectif de réduire la morbidité et la mortalité dues aux maladies évitables par la vaccination. II s'agit plus spécifiquement d'atteindre une couverture d'au moins 95\% d'ici 2016. Alors, nous avons constaté avec satisfaction la nette évolution du taux de couverture vaccinale enregistré par le 
Programme Elargi de Vaccination (PEV) qui est passé de $89 \%$ en 2009 à $90 \%$ en 2012 pour le vaccin contre la diphtérie, le tétanos, la coqueluche, l'haemophilus influenzae type b et l'hépatite b [6].

Malgré ces efforts et ces résultats satisfaisants, certains indicateurs de performance du PEV connaissent de plus en plus une stagnation voire même une régression. Cette situation préoccupante s'observe au niveau de la continuité du calendrier vaccinal où, le taux d'abandon entre la première et la troisième dose du vaccin pentavalent pose un problème réel de par son importance.

$\mathrm{Au}$ Mali, la couverture vaccinale en DTC3 (Pentavalent3) est passée de $68 \%$ en 2006 à $63 \%$ en 2012 avec respectivement des taux de déperdition de $19 \%$ et $21 \%$ entre la première et la troisième [7]. Selon les sources de DVD_MT 2015 de la section d'immunisation de la Direction Nationale de la Santé du Mali, le district sanitaire de la commune IV avait enregistré un taux d'abandon Penta1-Penta3 de 19\%. Ce taux dépasse le taux national qui doit être inférieur à $10 \%$.

Plusieurs facteurs pourraient expliqués cet état de fait, notamment ceux communautaires ont été les plus incriminés à travers plusieurs études. Certains auteurs trouvent le manque de temps (22\%) [8] , la non maitrise du calendrier vaccinal $(50,8 \%)$ [9] et le manque d'intérêt pour la vaccination (62\%) [10].

Au regard de ce qui précède, nous nous sommes proposés d'étudier les facteurs communautaires influençant la forte déperdition entre la première et la troisième dose du vaccin Pentavalent chez les enfants de 0 à 11 mois dans le district sanitaire de la commune IV de Bamako du 1er janvier au 31 décembre 2016 dans le but d'apporter des solutions à cette forte déperdition.

\section{Matériels et Méthodes}

Cadre de l'étude : Cette étude a été menée dans le district sanitaire de la commune IV de Bamako, comportant dix aires de santé avec une superficie de $37,68 \mathrm{Km}^{2}$ et une population estimé à 342516 habitants en 2016. La population cible du PEV des enfants de 0 à 11 mois était de 13701.

Type et période d'étude: il s'agissait d'étude transversale, descriptive et analytique concernant la période du 1er janvier au 31 décembre 2016.

Population d'étude : La population était constituée des enfants de 0 à 11 mois avec comme répondant leurs tuteurs, les chargés PEV des aires de santé et du Centre de Santé de Référence (CSRéf), les médecins Directeurs des aires de santé et le médecin chef du CSRéf.

Ont été inclus dans l'étude, les enfants nés entre le 1er décembre 2015 et le 15 septembre 2016, disposant d'un carnet de vaccination et ayant fait la première dose $\mathrm{du}$ vaccin Pentavalent.

N'ont pas été inclus, les enfants n'ayant pas utilisés les services de vaccination du district sanitaires de la Commune IV et les tuteurs d'enfant absents pendant la période de collecte des données ou ayant refusés de participer à l'enquête.

Echantillonnage : la détermination de la taille nécessaire à l'étude pour les tuteurs d'enfants a été calculé à partir de la formule de Schwartz $\left(n=z 2^{*} p^{*} q / i 2^{*} d\right)$. La taille maximale de l'échantillon a été de 380 sujets dont 360 tuteurs d'enfants et 20 agents de santé. La méthode probabiliste a été utilisée pour le choix des tuteurs d'enfants et non probabiliste pour le choix des agents de santé. Nous avons adopté le sondage en grappes à deux degrés pour repartir les 360 tuteurs dans 30 grappes (360/30) soit une moyenne de 12 enfants par grappe. Pour le choix des ménages à visiter, un repère a été choisi dans chaque aire de santé et à partir de ce repère, nous avons déterminé une direction au hasard en lançant un stylo en l'air et nous avons commencé l'enquête à partir de la direction indiquée par la pointe du stylo. Ainsi de proche en proche, nous avons parcouru l'aire de santé jusqu'à atteindre le nombre de grappe. Pour les agents, un choix raisonné nous a permis de les sélectionner pour l'entrevue.

Techniques et outils de collecte des données : les données ont été collectées à l'aide d'un questionnaire structuré préalablement testé. La collecte des données s'est déroulée du 11 au 20 janvier 2018 dans les aires de santé du district sanitaire de la commune IV et la collecte a consisté à l'administration des questionnaires aux différentes cibles de l'étude et l'exploitation des supports de gestion PEV.

Traitement et analyse des données : le traitement des données a été à la fois manuel et informatisé et fait l'objet d'une vérification des données et la correction des éventuelles erreurs d'enregistrement sur les fiches de collecte de données.

Les données ont été saisies dans une base de données faite par le logiciel Epi Info version 7 et les analyses effectuées à l'aide du logiciel SPSS version 21.0. Les contrôles de saisie nous ont permis de minimiser les erreurs. Une analyse bi-variée par régression logistique nous ont permis d'obtenir des odds ratio pour chacune des variables avec leurs intervalles de confiance à $95 \%$ et leurs valeurs $P$. Ensuite les variables ayant obtenu une valeur de $p<0,05$ ont été toutes entrées dans un modèle de régression logistique multi-variée afin d'identifier les facteurs associés au taux de déperdition Penta1-Penta3. Ainsi les variables ayant une valeur $P<0,05$ de ce modèle multi-variée avec des intervalles de confiance à $95 \%$ qui ne contiennent pas 1,00 étaient considérées comme statistiquement significatives et celles qui avaient obtenu dans ce modèle une valeur $P$ non significative $(>0,05)$, étaient sorties du modèle. De ce faite nous avons retenu un modèle final.

Considérations éthiques: les enquêtés ont été rassurés de la confidentialité et de l'anonymat. Un consentement éclairé des enquêtés a été d'abord obtenu 
avant le démarrage des entretiens. Ils ont été libres d'accepter ou de refuser avant ou pendant l'entretien.

\section{Résultats}

Caractéristiques démographiques de la population d'étude

Trois cent soixante (360) tuteurs d'enfants de 0 à 11 mois ont été inclues, leurs âge variait de 16 à 43 ans avec une moyenne de 27 ans et écart-type de 5,78. La classe d'âge la plus représentée était celle des 25 à 34 ans $(55,56 \%)$; seulement $8,61 \%$ étaient de sexe masculin avec un sex ratio de 0,09 en défaveur du sexe masculin ; les mariés dominaient avec $88,61 \%$; les non instruits ne représentaient que $20 \%$. L'âge des enfants enquêtés variait de 15 à 23 mois avec une moyenne de 19,67 et un écart-type de 2,781. Plus de $72 \%$ des enfants avaient un âge compris entre 18 et 23 mois. Le sexe masculin dominait $(51,39 \%)$ avec un sex ratio de 1,05 en faveur du sexe masculin pour les enfants (Tableau 1).

La déperdition Penta1-Penta3 chez les enfants de 0 à 11 mois

Sur les 360 enfants de 0 à 11 mois enquêtés, 88 n'avaient pas reçu la troisième dose du vaccin Pentavalent soit un taux de déperdition Pentavalent1Pentavalent 3 de $24,44 \%$.

A l'exception de l'aire de santé ASACOLAB5 (2,78\%), toutes les aires ont dépassé le taux national de déperdition Pentavalent1-Pentavalent3 qui doit être inférieur à $10 \%$

ASACOSEKASI et ASACOSEK ont enregistré plus de taux de déperdition avec respectivement $43,75 \%$ et $33,33 \%$ (Figure 1).

Les raisons de non vaccination évoquées par leurs tuteurs d'enfants

Quatre-vingt huit (88) tuteurs d'enfants de 0 à 11 incomplètement vaccinés ont évoqué comme raisons de non vaccination: les occupations $(29,55 \%)$, le déplacement $(15,90 \%)$ et le rendez-vous manqués $(14,77 \%)$ (Tableau 2).

Prédicteurs indépendants du taux de déperdition Pentavalent1-Pentavalent3

Nous avons trouvé que les tuteurs d'enfants de 0 à 11 mois du district sanitaire de la commune IV qui avaient la mauvaise connaissance des maladies cibles du Pentavalent $(p=0,002)$, la non connaissance des avantages de la vaccination ( $p=0,007)$, qui n'étaient pas disponibilité $(p=0,046)$ et qui appréciaient le temps d'attente long $(p=0,023)$ étaient statistiquement associés à la déperdition Pentavalent1-Pentavalent3.

Les dépenses effectuées $(p=0,2)$ et le nombre de séance de vaccination par semaine $(p=0,081)$ n'ont pas été associés à la déperdition Pentavalent1-Pentavalent3 (Tableau 3).

Discussion

Le taux de déperdition Pentavalent1-Pentavalent3 Le taux de déperdition est un indicateur de performance de la réduction des épidémies des maladies évitables par la vaccination et surtout de celle de la mortalité infantojuvénile.

Dans cette étude, il a été enregistré un taux de déperdition Pentavalent1-Pentavalent3 de $24,44 \%$ chez les enfants de 0 à 11 mois dans le district sanitaire de la commune IV en 2016. Or selon les normes nationale et internationale, ce taux doit être inférieur à 10\%. Au Mali l'Enquête Démographique et de Santé du Mali (EDSM V) 2012-2013 et l'enquête nationale de couverture vaccinale de routine et d'évaluation post campagne de vaccination contre la rougeole (ENCVA) 2015 trouvent respectivement $21 \%$ et $20,51 \%$ de taux de déperdition Pentavalent1-Pentavalent3 au plan national [11, 12]. Cette différence pourrait s'expliquer par des taux d'abandon très élevés dans deux grandes aires de santé aux périphéries de la commune IV (ASACOSEKASI et ASACOSEK) qui avaient respectivement $43,75 \%$ et $33,33 \%$.

Facteurs associés au taux de déperdition Penta1Penta3

Dans cette étude, les analyses multi-variées réalisées nous ont permis de mettre en évidence une association statistiquement significative entre la non connaissance des maladies cibles du Pentavalent $(p=0,000 ; O R=0,39$; $\left.\mathrm{IC}_{95 \%}[0,23-0,64]\right)$, la non connaissance des avantages de la vaccination $\left(p=0,000 ; O R=3,96 ; I_{95 \%}[2,15\right.$ $7,30])$, la non disponibilité du tuteur $(p=0,000 ; O R=0,22$; $\left.\mathrm{IC}_{5 \%}[0,11-0,44]\right)$ et le long temps d'attente $(p=0,023$; $\mathrm{OR}=0,495$; IC $95 \%[0,270$ - 0,906]) et le fort taux de déperdition Pentavalent1-Pentavalent3 en commune IV du District de Bamako. En effet, les enfants ayant des tuteurs ne connaissant pas les avantages de la vaccination, ont 4 fois plus de risque d'abandon.

Ces résultats corroborent avec ceux de Pouth SFB et col au Cameroun (2012), qui trouvent que la non connaissance de l'importance de la vaccination $\left(p=0,0139 ; I_{95 \%}[1,3514-14,4216]\right)$ et le délai d'attente de plus d'heure ( $p=0,0005 ;$ I $\mathrm{C}_{95 \%}$ [2,5329 - 27,7778]) sont statistiquement associés à l'incomplétude vaccinale des enfants de 12 à 23 mois [1].

Yohannes $A$ et al en Ethiopie (2017) révèlent que les participants qui avaient une connaissance élevée en vaccination étaient 2,24 fois plus susceptibles de vacciner leurs enfants que ceux qui avaient une faible connaissance $\left(\mathrm{OR}=2,24 ; \mathrm{IC}_{95 \%}[1,68-2,98]\right)[12]$.

En effet la connaissance des maladies cibles du Pentavalent par les tuteurs constitue le premier pilier vers le changement de comportement favorable à la vaccination, entrainant leur adhésion à l'immunisation de leurs enfants. Ce processus les amènera à appréhender les avantages de la vaccination. Ainsi, ils se rendront disponibles et accorderons plus de temps afin de vacciner correctement et complètement leurs enfants. Ceci est le fondement de l'assiduité des tuteurs à la complétude vaccinale de leurs enfants.

CONCLUSION

Cette étude portant sur les facteurs communautaires influençant la déperdition entre la première et la troisième 
dose du vaccin Pentavalent chez les enfants de 0 à 11 mois dans le district sanitaire de la commune IV en 2016, a permis de conclure que le taux de déperdition en Pentavalent1-Pentavalent3 constitue un réel problème de performance du PEV.

Au terme de notre étude, ce taux a été associé à la non connaissance des avantages de la vaccination, la non connaissance des maladies cibles du Pentavalent, le long temps d'attente et la non disponibilité des tuteurs. Pour l'améliorer, la mise à l'échelle de la stratégie «Atteindre Chaque District (ACD) », « Atteindre Chaque Enfant (ACE) », l'information et la sensibilisation de la communauté sur les bienfaits et les avantages de la vaccination, le raccourcissement du temps d'attente et le respect du calendrier vaccinal sont nécessaires.

\section{Références bibliographiques:}

1. Ba Pouth SFB, Kazambu D, Delissaint D, Kobela M. Couverture vaccinale et facteurs associés à la non complétude vaccinale des enfants de 12 à 23 mois du district de santé de Djoungolo-Cameroun en 2012. Pan Afr Med J. 4 févr 2014;17:1-7 p.

2. OMS | Programmes et systèmes nationaux [Internet]. WHO. [cité 1 nov 2017]. Disponible sur: http://www.who.int/immunization/programmes_systems/fr

3. OMS | Couverture vaccinale [Internet]. WHO. [cité 29 oct 2017]. Disponible sur: http://www.who.int/mediacentre/factsheets/fs378/fr/ 4. OMS / Couverture par la vaccination systématique dans le monde 2011 [Internet]. WHO. [cité 1 nov 2017]. Disponible sur: http://www.who.int/immunization/documents/vaccination coverage/fr/

5. OMS/UNICEF. Communiqué de presse conjoint_Un enfant sur 10 dans le monde n'a reçu aucun vaccin en 2016 [Internet]. WHO. [cité 1 nov 2017]. Disponible sur:

http://www.who.int/mediacentre/news/releases/2017/infa nts-worldwide-vaccinations/fr/
6. MSHP-Mali, Direction Nationale de Santé/Section d'Immunisation. Plan pluriannuel complet révisé de la vaccination 2012-2016. 173 p.

7. MS-Mali, Cellule de Planification et de Statistique, Direction Nationale de la Statistique et de I'Informatique, Macro International Inc. Enquête Démographique et de Santé du Mali (EDSM-IV) 2006. $497 p$.

8. Félicité Emma YAGATA MOUSSA. Étude des causes de faibles couvertures vaccinales dans la circonscription sanitaire du quatrième arrondissement de Bangui en Centrafrique, 2008 | EpivacPlus. 44 p. 2008. 9. Comlan DANDEGLA HOUILEY. Analyse comparée des taux d'abandon de la vaccination entre le BCG et le VAR dans les communautés partenaires et non partenaires de l'ONG Plan Bénin dans la commune de Klouékanmè au Bénin au 31 décembre 2006 | EpivacPlus. $55 \mathrm{p}$.

10. MSHP-Mali/Centre National d'Information, d'Education et de Communication Pour la Santé (CNIECS), UNICEF, OMS, GAVI, USAID, Coopération canadienne, Rotary international. Plan stratégique national de communication du Programme Elargi de Vaccination 2016 - 2019. 47 p.

11. MS-Mali, Cellule de Planification et de Statistiques, Institut National de la Statistique, Centre d'Études et d'Information Statistiques, ICF International. Enquête Démographique et de Santé du Mali (EDSM-V) 2012-2013. 547 p.

12. Yohannes A, Fiona B, Aschalew T, Kinde G, Tefera T, Yohannes M, et al. Behavioral determinants of immunization service utilization in Ethiopia: a crosssectional community-based survey. Pan Afr Med J. 2017;27(Suppl 2):1-6 p.

13. MSHP-Mali, Direction Nationale de la Santé/Institut National de la Statistique. Enquête nationale de couverture vaccinale de routine et d'évaluation post campagne de vaccination contre la rougeole (ENCVA) 2015. Rapport d'analyse. 72 p. 2016. 
Tableau I : Répartition des tuteurs selon certaines caractéristiques démographiques dans le district sanitaire de la commune IV en 2016.

\begin{tabular}{|c|c|c|}
\hline Caractéristiques démographiques & Effectifs $(n=360)$ & Pourcentage \\
\hline \multicolumn{3}{|l|}{ Tranche d'âge des enfants (mois) } \\
\hline 15 à 17 & 100 & 27,78 \\
\hline 18 à 20 & 87 & 24,17 \\
\hline 21 à 23 & 173 & 48,05 \\
\hline \multicolumn{3}{|l|}{ Tranche d'âge des tuteurs (années) } \\
\hline 15 à 24 & 116 & 32,22 \\
\hline 25 à 34 & 200 & 55,56 \\
\hline 35 à 44 & 44 & 12,22 \\
\hline \multicolumn{3}{|l|}{ Sexe des enfants } \\
\hline Féminin & 175 & 48,61 \\
\hline Masculin & 185 & 51,39 \\
\hline \multicolumn{3}{|l|}{ Sexe des tuteurs } \\
\hline Féminin & 329 & 91,39 \\
\hline Masculin & 31 & 8,61 \\
\hline \multicolumn{3}{|l|}{ Situation matrimoniale } \\
\hline Célibataire & 37 & 10,28 \\
\hline Divorcé(e) & 3 & 0,83 \\
\hline Marié(e) & 319 & 88,61 \\
\hline Veuf (ve) & 1 & 0,28 \\
\hline \multicolumn{3}{|l|}{ Niveau d'instruction } \\
\hline Non instruit(e) & 72 & 20,00 \\
\hline Instruit(e) primaire & 139 & 38,61 \\
\hline Instruit(e) secondaire & 110 & 30,56 \\
\hline Instruit(e) supérieur & 39 & 10,83 \\
\hline
\end{tabular}

Tableau II : Raisons de non vaccinations avec le Pentavalent évoquées par les tuteurs

\begin{tabular}{lcc}
\hline Raisons de non vaccination & Effectifs n=88 & Pourcentage \\
\hline Beaucoup d'injections en une seule fois & 1 & 1,14 \\
Déplacement du tuteur & 14 & 15,90 \\
Occupation du tuteur & 26 & 29,55 \\
Maladie du tuteur & 1 & 1,14 \\
Rendez-vous manqués par le tuteur & 13 & 14,77 \\
Refus du père & 6 & 6,82 \\
Indisponibilité du vaccin & 1 & 1,14 \\
Maladie de l'enfant & 10 & 11,36 \\
Pas nécessaire de revenir pour la 3ème dose suivante & 12 & 13,64 \\
Peur des réactions secondaires & 4 & 4,54 \\
\hline
\end{tabular}


Tableau III : Facteurs associés au taux de déperdition Penta1-Penta3 des enfants de 0 à 11 mois du district sanitaire de la commune IV en 2016 (Résultat de la régression logistique multi-variée)

\begin{tabular}{|c|c|c|c|c|}
\hline \multirow[t]{2}{*}{ Variables } & \multirow{2}{*}{ P-value } & \multirow{2}{*}{ OR } & \multicolumn{2}{|c|}{$\mathrm{IC}_{95 \%}$} \\
\hline & & & Inférieur & Supérieur \\
\hline $\begin{array}{l}\text { Dépenses effectuées } \\
\text { (moins de } 1200 \text { Fcfa/ } 1200 \text { Fcfa et plus) }\end{array}$ & 0,200 & 0,616 & 0,293 & 1,292 \\
\hline $\begin{array}{l}\text { Nombre de séance de vaccination par semaine } \\
\text { (moins de } 3 \text { jours/ } 3 \text { à } 5 \text { jours) }\end{array}$ & 0,081 & 0,574 & 0,308 & 1,071 \\
\hline $\begin{array}{l}\text { Connaissance des avantages de la vaccination } \\
\text { (Non/ Oui) }\end{array}$ & 0,007 & 2,699 & 1,304 & 5,589 \\
\hline $\begin{array}{l}\text { Connaissance des maladies cibles du Penta } \\
\text { (mauvaise/ bonne) }\end{array}$ & 0,002 & 0,386 & 0,209 & 0,716 \\
\hline $\begin{array}{l}\text { Appréciation temps d'attente } \\
\text { (acceptable/ long) }\end{array}$ & 0,023 & 0,495 & 0,270 & 0,906 \\
\hline $\begin{array}{l}\text { Disponibilité du tuteur } \\
\text { (disponible/ non disponible) }\end{array}$ & 0,046 & 0,423 & 0,182 & 0,983 \\
\hline
\end{tabular}

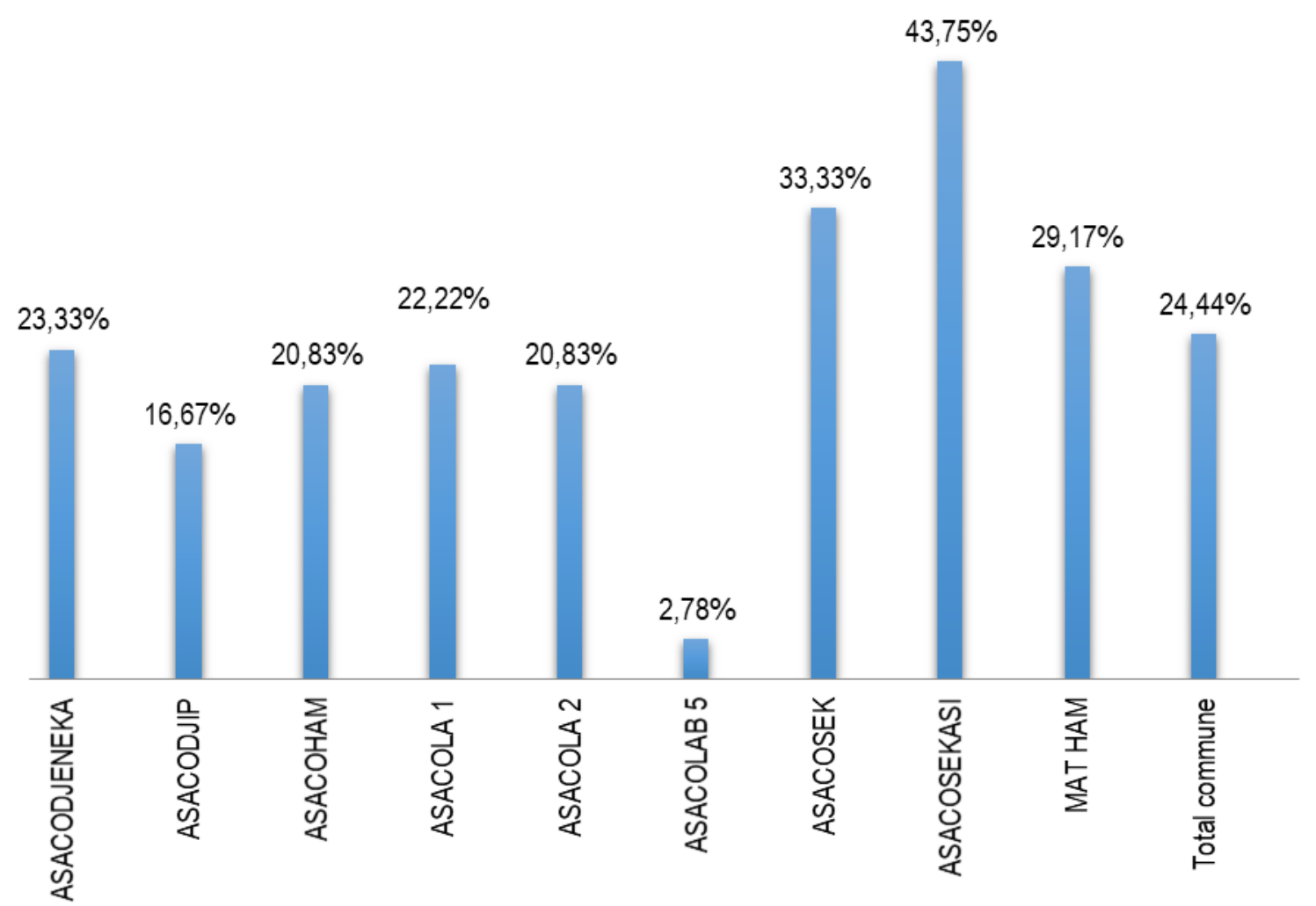

Figure 1 : Evolution du taux de déperdition Penta1- Penta3 en fonction des aires de santé chez les enfants de 0 à 11 mois dans le district sanitaire de la commune IV en 2016. 